\title{
Delimitación de áreas sociales en el Barrio de la Concepción. Un ensayo de ecología factorial a escala microespacial.
}

\author{
Gloria lora-Tamayo D'ocon* y Félix Aparicio Pérez**
}

\section{INTRODUCCIÓN}

Desde que en 1965 F.L. Sweester realizará sobre la ciudad de Helsinki el primer análisis de Ecología Factorial -entendiendo como tal, la aplicación de las técnicas de Análisis Factorial a los datos que describen la diferenciación residencial de la población-, se han realizado numerosos estudios de ciudades, sobre todo americanas, dentro de este campo.

Los análisis de Ecología Factorial aplicados a ciudades han respaldado, en términos generales, la hipótesis del Modelo de Áreas Sociales de E. Shevky y W. Bell (1955), poniendo de relieve, como factores fundamentales de diferenciación social, el Status Económico o Rango Social y el Status Familiar, y como factores secundarios, que no aparecen en muchos casos, el Status Étnico y la Movilidad de la Población.

El caso de la ciudad de Madrid ha ocupado en los últimos años la atención de sociólogos y geógrafos, siendo varios los estudios de Ecología Factorial aparecidos hasta el momento (LORA TAMAYO, G. 1990).

\footnotetext{
* Colaborador H. Dpto. Análisis Geográfico Regional y Geografia Fisica. UCM.

** Estadístico. Facultativo INE.
} 
Todos ellos toman como unidad de análisis los barrios municipales de la ciudad, si bien el último realizado por JIMENEZ BLASCO, B.C. (1986), incluye análisis también en base a unidades de escala mayor, como son los distritos, y menor, como las secciones censales. Estas últimas son, asimismo, las unidades escogidas por AGUILERA ARILLA, M.J., (1988) en su análisis de Ecología Factorial del municipio de Alcorcón, dentro del área metropolitana de Madrid.

La fuente básica de donde se extraen las variables introducidas en estos análisis es el Padrón Municipal de Habitantes de 1975, bien directamente, a través de las hojas de inscripción familiar, bien indirectamente, a través de la explotación realizada por COPLACO de la actualización padronal de 1977.

En cuanto a los resultados, parecen ser el Status Social, la Edad y el grado de Urbanización-Familismo, los factores más potentes en la diferenciación espacial de la población de la ciudad de Madrid. El primero es el factor más claro, explica el mayor porcentaje de varianza y se relaciona con variables muy concretas (cuadros superiores y niveles de instrucción elevados, frente a niveles de instrucción bajos y trabajadores manuales). La Edad o grado de envejecimiento-juventud de la población, contrapone la población joven a la población anciana. Por último, JIMENEZ BLASCO, B.C., detecta un factor Urbanización (1986) opuesto al factor Familismo (misma autora, 1984), que aparece con mayor o menor fuerza en los distintos análisis factoriales llevados a cabo y en algunos casos se relaciona estrechamente con el factor Edad. El factor contrapone las variables personas casadas, tasa de fecundidad elevada, mujeres dedicadas a las labores del hogar, grupos de edades jóvenes, menores y estudiantes, a personas solteras o viudas, ancianos, hogares con uno o dos ocupantes, etc

El presente estudio constituye un ensayo de Ecología Factorial, referido a un barrio concreto de la ciudad de Madrid. Los objetivos que se persiguen son:

1) Descender un peldaño en la unidad de análisis trabajando a nivel de manzana urbana, con objeto de ver si es posible llegar a una estructura factorial coherente en esta escala de desagregación espacial.

2) Detectar cuáles son los factores de diferenciación sociodemográfica en el barrio y si son coincidentes con los hallados para Madrid en otros estudios de Ecologia Factorial.

3) Comprobar si existen áreas sociales diferenciadas entre sí y si éstas tienen alguna relación con las áreas morfológicas en que se puede distribuir el espacio de estudio. 
La elección del Barrio de la Concepción como objeto de análisis se debe, sobre todo, a su función residencial y a los importantes contrastes que a primera vista encierra.

La fecha de referencia es 1975 . Con ello queremos hacer nuestros resultados comparables con los de los otros estudios referidos a Madrid. La fuente empleada ha sido el Padrón Municipal de Habitantes, explotado y elaborado personalmente al $100 \%$ de las hojas, a nivel de manzana.

En cuanto a la metodología, empleamos el Análisis Factorial en sus modalidades de Factores Principales y Máxima Verosimilitud.

\section{EL ESPACIO DE ESTUDIO}

El Barrio de la Concepción se sitúa en el cuadrante Nororiental de Madrid, formando parte de la periferia próxima de la ciudad y desarrollándose transversalmente entre la autopista $M-30$, al Oeste, y el limite oriental de la Ciudad Lineal, al Este, y entre los barrios de San Pascual, al Norte, y Quintana, al Sur, que constituyen, con el de la Concepción, el Sector Central del Distrito de Ciudad Lineal (fig. 1).

Las 90 Has que encierra el barrio no son un continuo urbano uniforme, sino que, como consecuencia de su dilatada formación en el tiempo, el espacio se halla compartimentado en una serie de sectores perfectamente diferenciables desde el punto de vista morfológico y funcional.

De éstos, los que mayor desarrollo espacial tienen y mayor volumen de población albergan son las promociones de viviendas de ala Concepción" y su "Ampliación».

La primera se sitúa en el centro del área de estudio. Se trata de un espacio residencial morfológicamente homogéneo, en la base del cual se extiende un amplio parque -el Parque Calero- y un Polideportivo. Data de los años 50 y fue construida por la empresa Banús, como respuesta a la fuerte demanda de viviendas que se produjo en Madrid, consecuencia de la corriente inmigratoria hacia la ciudad y el importante crecimiento vegetativo registrado en la época. La Ampliación, diez años más joven, se encuentra al Oeste de la Concepción; también es un área residencial morfológicamente homogénea -fruto de otro proyecto unitario-, que está formada por grandes bloques residenciales de entre 11 y 16 plantas. Por su magnitud, ambas promociones han albergado desde su creación a la mayor parte de la población del barrio (en torno al $70 \%$ ). 


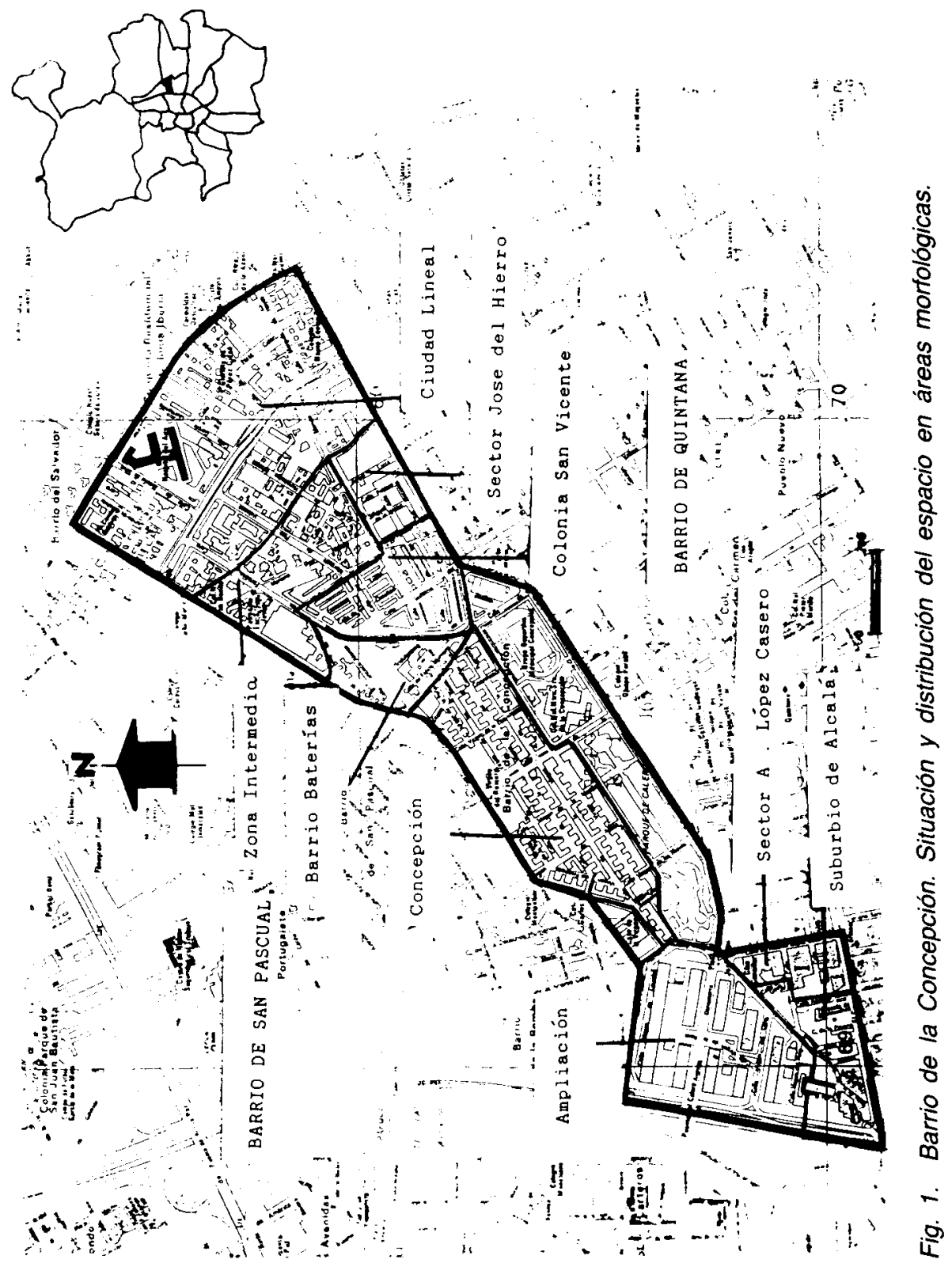


Al pie de la Ampliación se encuentra una de las zonas más antiguas del barrio, situada de cara a la calle Alcalá, eje de crecimiento de importancia singular dentro de Madrid. Esta zona, que data de finales del siglo pasado y principios del presente, ha sufrido remodelaciones eficaces únicamente en las manzanas más orientales, limítrofes al barrio de Quintana - de cara a la calle Alcalde López Casero-, que han sido las únicas que se han salvado del destino de expropiación y demolición establecido por el Plan Especial Avenida de la Paz, para las manzanas edificadas al borde del antiguo arroyo Abroñigal, de antigua creación y mal estado de conservación. Este área concreta fue degradándose progresivamente desde mediados de los años 60 hasta desaparecer totalmente bajo la piqueta al inicio de los 80 . Hoy en día es un solar, al Norte del cual aún queda en pie el antiguo Mercado de Canillas, en la actualidad "Centro Comercial Las Ventas", y las tres manzanas remodeladas que comentamos anteriormente, de cara a la calle Alcalde López Casero.

Al Este de la promoción de la Concepción, el barrio se abre ligeramente en abanico hacia la Ciudad Lineal. El área es también fundamentalmente residencial, si bien la ocupación del suelo es sobre todo extensiva, salvo en el caso de una amplia manzana, al Norte, de uso industrial, y un grupo de pequeñas manzanas, al Sur, de cara a la calle José del Hierro - límite del barrio en este sector- de edificación cerrada y uso mixto residencial e industrial. Esta última zona, urbanizada en los años 60 , es fruto de un proyecto específico y cuenta con un cierto número de viviendas de protección oficial, asi como otras de anterior construcción, englobadas en el conjunto.

Al Oeste del "Sector José del Hierro" y también de cara a esta calle y al Polideportivo Municipal, destaca un área homogénea de chalecitos adosados, la Colonia San Vicente, que nació a finales de los años 40 y ha ido desarrollándose en sucesivas fases, concluyendo su perimetro en los años 80. A pesar de su largo proceso de formación, la colonia tiene unidad y personalidad propias, destacando en el entorno.

Al Noroeste de ella, el "Barrio Baterías" se encaja a modo de cuña, entre la Colonia San Vicente y la Concepción. Se trata de un suburbio constituido sobre todo por edificios unifamiliares, en su mayoria de baja calidad, mal estado de conservación o ruina, muchos de los cuales datan de principios de siglo. Entre los solares y calles sin asfaltar ni pavimentar, proliferaron las chabolas hasta fines de los años 70 . Muchas de las viviendas no cuentan con los servicios mínimos. La única excepción en el área es un bloque multifamiliar aislado, en edificación cerrada, de cara a la calle Estrecho de Corea, construido al inicio de los años 60, fruto de un proyecto más ambicioso, que no llegó nunca a realizarse. 
El extremo oriental del barrio está ocupado por un tramo de la Ciudad Lineal, proyectada a principios de siglo por Arturo Soria, que en este sector nunca llegó a rellenar todo su territorio. Sí quedan señaladas en el plano las amplias manzanas a lo largo de la calle central, calle Arturo Soria, remodelada en los años 70 según un Plan Parcial que engloba lo que hoy queda del proyecto primitivo. Es un área poco homogénea, a pesar de haber nacido de un plan unitario, contándose en ella hoteles de lujo, burgueses y obreros de principios del siglo, en distinto estado de conservación; viviendas marginales, producto de una ocupación individual y espontánea del espacio -testigos de una época en que la Ciudad Lineal quedó abandonada a su suerte-; chalets modernos, construidos a partir de los años 60 y bloques multifamiliares en edificación abierta, de hasta cuatro alturas, levantados también a partir de la misma fecha. Este es el tipo residencial con el que en la actualidad se rellena el suelo vacante, como expresión del Plan Parcial de Ordenación de la Ciudad Lineal. La fuerte revalorización del terreno en los últimos años, ha tenido como consecuencia la mejora en la calidad arquitectónica de los edificios y de las instalaciones y servicios con que éstos cuentan (jardines, piscinas, canchas de tenis...), tendiendo a configurarse la Ciudad Lineal como un área residencial de alto standing.

Entre la Ciudad Lineal, el Sector José del Hierro y la Colonia San Vicente, se extienden, por último, unas manzanas que no configuran un área unitaria. La ocupación del suelo es extensiva en general, combinándose edificios unifamiliares al estilo de la colonia San Vicente o de la Ciudad Lineal, con viviendas marginales, bloques multifamiliares en edificación cerrada de distintas épocas y otros multifamiliares abiertos, algunos de los cuales son de viviendas de lujo. El área no está colmatada, abundando los solares. Por su carácter ecléctico y transicional entre áreas de fuerte personalidad, la denominamos Zona Intermedia.

La población del barrio alcanzó los valores más elevados en 1970, en que se contabilizaron 31.589 personas residentes. A partir de esa fecha, se observa una continua disminución, como efecto del progresivo envejecimiento, el descenso en las tasas de natalidad y fecundidad y la emigración fuera del barrio de parte de la población joven. El volumen de residentes pasa a 28.361 en 1975, 26.281 en 1981 y 24.264 en 1986. Su distribución espacial, sin embargo, permanece bastante estable: las áreas de edificación cerrada de la Ampliación, la Concepción, José del Hierro y Alcalde López Casero, aglutinan una cifra en torno al $85 \%$ de la población total, correspondiendo a las restantes zonas, todas de edificación abierta, el $15 \%$ restante. La tendencia actual es a una ligera pérdida de peso de las primeras zonas en favor de las segundas, en 
función del envejecimiento de la trama urbana de aquellas y la intensa dinámica constructiva en éstas, sobre todo en la Ciudad Lineal y la Zona Intermedia.

El Barrio de la Concepción es un mosaico urbano a escala microespacial. La compartimentación del espacio en áreas bien diferenciadas y la existencia de profundos contrastes residenciales otorga al espacio un interés singular como objeto de análisis, desde un punto de vista sociodemográfico.

\section{FACTORES DE DIFERENCIACIÓN SOCIODEMOGRÁFICA}

\subsection{Metodología}

En la fig. 2 se muestra la delimitación de las unidades de observación. El reducido volumen de población con que algunas manzanas cuentan, resta fiabilidad estadística a los valores de muchas de las variables consideradas. Para resolver el problema se adoptó la solución de unir manzanas próximas - dentro de una misma área morfológica-, en orden a obtener unidades de observación con un volumen mínimo de 50 habitantes. Se excluyeron, por otra parte, aquellas manzanas sin población o con un volumen de población pequeño y cuyo papel fundamental era el de «islas funcionales" '; de Este a Oeste, son: el Hospital del Aire, Coiegio San Benito, manzana industrial, Polideportivo y Parque Calero, Casa de la Virgen y Centro Comercial Las Ventas. El total de unidades de observación es de 85.

En un análisis previo se seleccionaron 23 variables sociodemográficas, relacionadas con el tipo de ocupación de la población, nivel de instrucción, caracteristicas demográficas y otros aspectos relativos al tipo de hogar/familia, el origen de la población y el status social. Las correlaciones entre las variables eran en general bajas, debido, sobre todo, al reducido volumen de población de las observaciones. Por su parte, la

' Tal como se definen en Gutierrez Ronco, S., Lora-Tamayo, G., y Casas Torres, J. M., 1977-78: "Materiales para el estudio de la población, abastecimiento y equipado de Carabanchel Alto en 1976", Geographica 1977-1978. Madrid, Instituto de Geografia Aplicada, CSIC, págs. 219-356. 

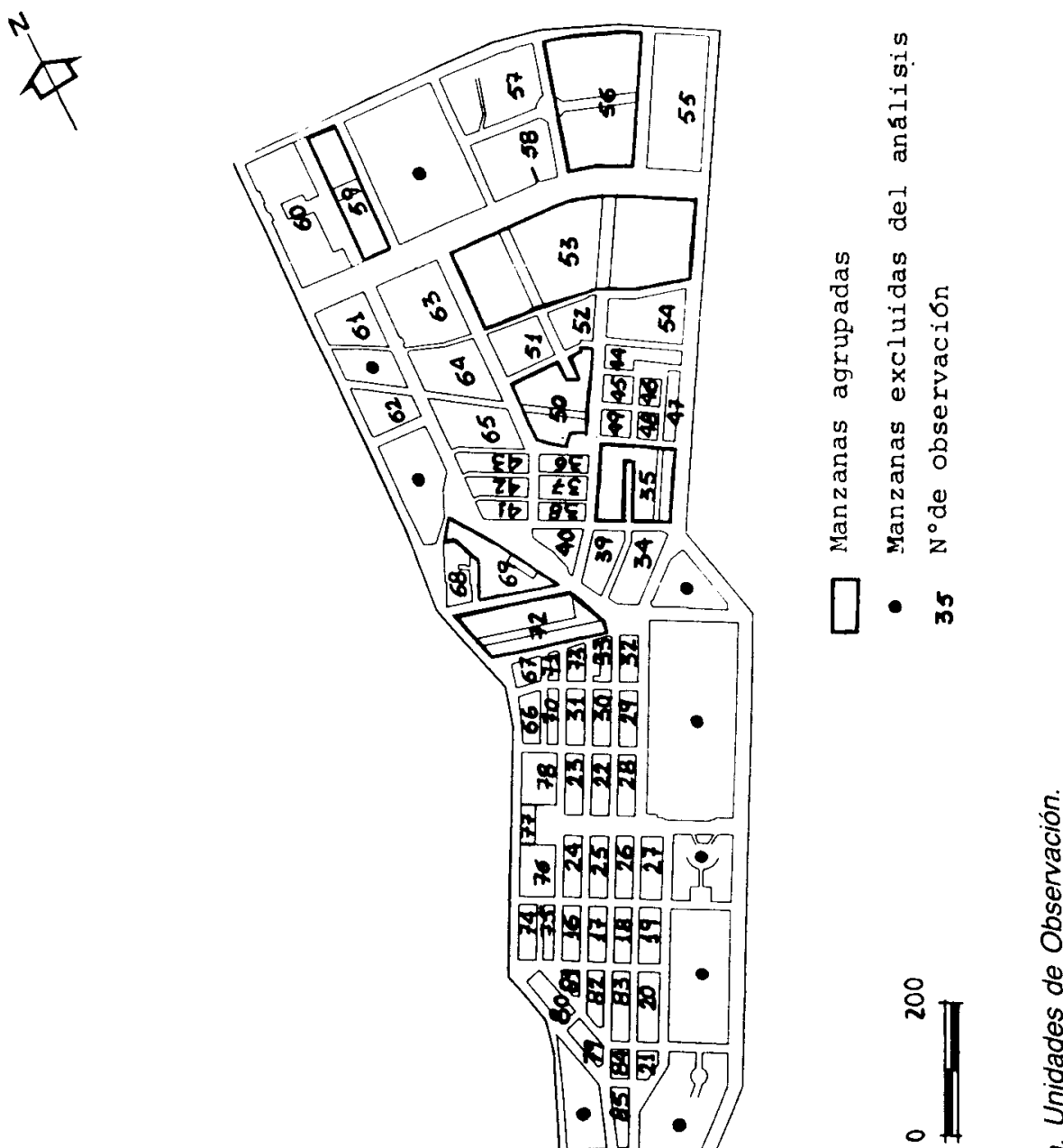
medida "Kaiser" de adecuación de las variables al modelo factorial, aplicada a la matriz de entrada, no destacó ninguna como óptima ${ }^{2}$.

Con todo, se ensayó un Análisis Factorial por el modelo del Factor Principal, del que resultó una matriz compuesta por ocho factores que explicaban en total el $56,26 \%$ de la varianza. Una vez rotada, por el método de Harris-Kaiser, de rotación oblicua, se obtuvieron ocho factores de los que cuatro estaban intercorrelados, siendo los más significativos, dos relacionados con el Status Social, dos relacionados con el grado de Urbanización-Familismo y un quinto factor de contenido demográfico. Entre todos explicaban el $69,72 \%$ de la varianza. Los tres factores restantes no tenian significación.

Excluidas seis de las variables que demostraron ajustarse peor al modelo, se realizó un segundo ensayo de Análisis Factorial, por el método de Factores Principales. En este caso, la matriz factorial obtenida, sin rotar, constaba de seis factores, que explicaban el $60 \%$ de la varianza total. La matriz factorial rotada, por el método de Harris-Kaiser, daba como resultado seis factores oblicuos, relacionados con los ejes Status Social (cuatro de ellos) y Urbanización (los dos restantes). Por el método de rotación Varimax, se obtuvo una estructura factorial bastante coherente, con seis factores ortogonales, de los que dos se relacionaban con el Status Social, dos con el Status Familiar y dos no eran significativos. El peso de los dos ejes era parecido, explicando entre ambos casi el $50 \%$ de la varianza total.

No presentamos aqui los resultados de los dos factoriales expuestos, por no considerarlos concluyentes.

En nuestra búsqueda de una estructura factorial clara, representativa y lo más simple posible, realizamos un nuevo Análisis Factorial, que consideramos definitivo, en el que se excluyeron de la primera matriz de datos, todas aquellas variables que consideramos poco adecuadas, bien por sus bajos coeficientes de correlación con las restantes, bien por sus reducidos valores, según la medida "Kaiser» de adecuación al modelo.

\subsection{Variables analizadas}

La matriz factorial de partida recoge las siguientes variables:

1) Patronos con asalariados (\% patronos, empresarios o profesionales que emplean personal, sobre la Población Total).

${ }^{2}$ Se consideran óptimas las que tienen un valor "Kaiser's Measure of Sampling Adequacy" superior a 0,80 y pésimas las que no alcanzan 0,40 . 
2) Profesionales técnicos y asimilados (\% de éstos sobre la P.T.).

3) Directivos (\% personal directivo de la administración pública y de las empresas, sobre la P.T.).

4) Trabajadores manuales ( $\%$ de trabajadores de la industria y de la construcción, no jefes ni supervisores, sobre la P.T.).

5) Analfabetos (\% personas de más de 10 años que no saben leer ni escribir, o tienen primaria incompleta, sobre la P.T.).

6) Bachillerato Superior (\% personas de más de 20 años cuyo nivel máximo de instrucción es Bachillerato Superior, sobre la P.T.)

7) Titulados Superiores y Medios (\% titulados superiores y medios sobre la P.T.).

8) Servicio Doméstico (\% hogares con servicio doméstico, sobre el total de hogares).

9) Menores de 15 años (\% menores de 15 años, sobre la P.T.).

10) Hogares sin núcleo (\% hogares sin núcleo familiar, sobre el total de hogares).

11) Familias Amplias (\% de familias de más de 5 componentes, sobre el total de hogares).

12) Mujeres Activas (\% mujeres activas, sobre el total de mujeres).

Se echa de menos en el listado la inclusión de variables relacionadas con el estado civil de la población y alguna más relacionada con la edad; sin embargo, ambos tipos de variables han demostrado adecuarse mal al modelo factorial aplicado al Barrio de la Concepción.

La matriz de correlaciones (Cuadro I) ofrece, sobre todo, valores medios y bajos, debido al nivel de desagregación en que operamos. Destacan con valores altos positivos, los índices de correlación entre las variables trabajadores manuales y analfabetos $(0,77)$ y profesionales técnicos y titulados superiores y medios $(0,74)$. Con valores medios positivos, los patronos con asalariados y directivos $(0,51)$, bachillerato superior y titulados superiores $(0,45)$, titulados superiores y servicio doméstico $(0,42)$ y hogares sin núcleo y mujeres activas $(0,41)$. Con valores medios negativos, destacan, por su parte, los indices de correlación entre las variables: trabajadores manuales y bachillerato superior $(-0,65)$, hogares sin núcleo y familias amplias $(-0,58)$, analfabetos y bachillerato superior $(-0,57)$, analfabetos y titulados superiores $(-0,54)$, analfabetos y profesionales técnicos $(-0,46)$, trabajadores manuales y titulados superiores $(-0,44)$ y familias amplias y mujeres activas $(-0,43)$. 
Delimitación de áreas sociales en el Barrio de la Concepción. Un ensayo ...

CUADRO I. MATRIZ DE CORRELACIONES
(1)
(2)
(3)
(4)
(5)
(6) (7)
(8)
(9) (10)
(11)
(12)

1) 1,00

2) $0,24 \quad 1,00$

3) $\quad 0,51 \quad 0,12 \quad 1,00$

4) $-0,18-0,32-0,40 \quad 1,00$

5) $\quad-0,23-0,46-0,33 \quad 0,77 \quad 1.00$

6) $\quad 0,09 \quad 0,28 \quad 0,19-0,65-0,57 \quad 1,00$

7) $\quad 0,35 \quad 0,74 \quad 0,28-0,44-0,54 \quad 0,45 \quad 1,00$

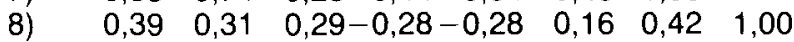

9) $\quad \begin{array}{lllllll}0.09 & 0,11 & 0,21-0,03-0,07 & -0,28 & 0,11 & 0,05 & 1,00\end{array}$

10) $-0,12 \quad 0,06-0,25 \quad 0,25 \quad 0,18-0,07-0,06-0,09-0,31 \quad 1,00$

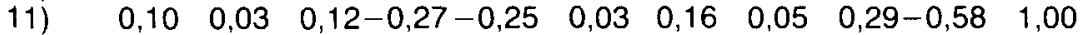

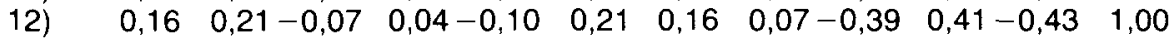

\section{MATRIZ FACTORIAL}

4. Trab. manuales

$-0,89$

5. Analfabetos

$-0,83$

7. Titulados sup-med.

0,72

6. Bto. Superior

0,68

2. Prof. Técnicos

0,56

3. Directivos

0,42

8. Servicio Doméstico

0,39

1. Patronos con asal

0,31

11. Familias Amplias

0,27

12. Mujeres activas

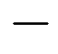

10. Hogares sin núcleo

-

9. Menores de 15 años

$\begin{array}{lc}0,33 & - \\ \overline{0,55} & - \\ \overline{0} & -\overline{0,38} \\ \overline{-} 57 & - \\ \overline{-} & - \\ -0,30 & - \\ 0,40 & - \\ 0,37 & -0,57 \\ - & -0,59 \\ & 0,57\end{array}$


MATRIZ FACTORIAL ROTADA (Método Harris-Kaiser)

10. Hogares sin núcleo ...... 0,73

11. Familias amplias ......... $-0,68$

12. Mujeres activas .......... 0,65

9. Menores de 15 años ..... $-0,54$

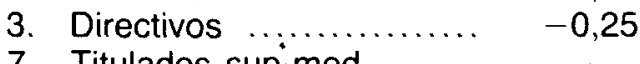

7. Titulados sup-med.

2. Profesionales técnicos

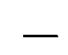

8. Servicio Doméstico

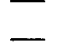

1. Patronos con asal.

5. Analfabetos ...............

4. Trabajadores manual. ....

6. Bachillerato sup.

(Los valores inferiores a 0,25 aparecen como -)

\subsection{Factores resultantes}

El método de Análisis Factorial empleado esta vez es el de Máxima Verosimilitud, que consiste en la obtención de una solución factorial que maximice la función de verosimilitud para la muestra dada (la función de verosimilitud es función de los parámetros, aunque coincide formalmente con la función de densidad de la muestra).

La matriz factorial resultante se compone de tres factores, entre los cuales explican el $51,01 \%$ de la varianza total. De ellos, el primero es el de mayor peso y significación, tratándose claramente de un factor de status social. Los otros dos factores resultan más difícilmente interpretables.

La matriz factorial rotada según el método de Harris-Kaiser, muestra una estructura clara y coherente: se resuelve en tres factores, de los cuales, el primero tiene relación con el Status Familiar o Urbanización, incluyendo las variables hogares sin núcleo y mujeres activas, que se contraponen a las familias amplias y los menores de quince años (estas últimas, con signo negativo). 
Por su parte, los factores 2 y 3 tienen relación con el Status social: el Factor 2 agrupa las variables de signo positivo, titulados superiores y medios, profesionales técnicos y asimilados, servicio doméstico y patronos con asalariados, $y$ las de signo negativo, analfabetos y trabajadores manuales (esta última con una carga baja). Es claramente un factor que se puede etiquetar como Alto Status Social.

El Factor 3 es de signo contrario: incluye cargas positivas en los trabajadores manuales y analfabetos y negativas en el nivel de bachillerato superior y titulados superiores (además de, con valores bajos, los directivos y profesionales técnicos y asimilados). Es, por tanto, un factor de Bajo Status Social.

El Factor 1 explica el $16,06 \%$ de la varianza; el 2 , el $21,59 \%$ y el 3 , el $22,32 \%$. En total, los tres factores explican el $59,97 \%$ de la varianza, correspondiéndoles al eje Status Social la mayor parte $(43,91 \%)$, consecuencia de que la mayoría de las variables de entrada seleccionadas forman parte de este eje.

En cuanto a la relación entre los factores resultantes, los dos relacionados con el Status Social están correlados negativamente, si bien el valor del índice no es elevado:

\section{CORRELACIÓN ENTRE LOS FACTORES:}

\begin{tabular}{lrrrr}
\hline & & F. 1 & F. 2 & F. 3 \\
\hline F. 1 & $\ldots \ldots \ldots$ & 1,00 & & \\
F. 2 & $\ldots \ldots \ldots$ & $-0,15$ & 1,00 & \\
F. 3 & $\ldots \ldots \ldots$ & 0,08 & $-0,40$ & 1,00 \\
\hline
\end{tabular}

En definitiva, los factores básicos que resumen la diferenciación sociodemográfica del Barrio de la Concepción, son dos: Status Social y Urbanización, factores invariantes que han ido apareciendo en sucesivos análisis, aplicando distintas técnicas de Análisis Factorial y empleando un número diferente de variables.

El eje Status Social, que explica la mayor parte de la varianza, está compuesto por las variables clásicas, si bien, al estar desdoblado en dos, reúne en un factor las variables características de alto statuts, y en otro las de bajo status. A lo largo de los distintos análisis llevados a cabo, la estructura del eje ha sido siempre la misma, modificándose únicamente 
el porcentaje de varianza explicada por el mismo, en un sentido ascedente, debido a que el peso de las variables relacionadas con él ha aumentado proporcionalmente, en las últimas matrices de entrada de datos.

Por su parte, el eje Status Familiar o Urbanización, que inicialmente aparecia desdoblado, se ha reducido a un único factor, que ha ido perdiendo significación dentro de la estructura factorial del barrio, por la razón contraria. Este factor, sin embargo, es muy puro y no se liga, como ocurría en la estructura de Madrid (JIMÉNEZ BLASCO, B. C. 1988), con variables indicativas de edad o estadio dentro del ciclo familiar.

El eje Edad o Envejecimiento/Juventud no aparece de manera significativa en nuestro barrio, aplicando el modelo factorial. Ello no quiere decir que no sea un factor diferenciador básico de las características residenciales del barrio, sino que, en nuestro caso, no ha resultado adecuado al modelo factorial. El hecho puede deberse a dos causas: que las variables de entrada no hayan sido bien seleccionadas o no hubiera un número suficiente de ellas relacionadas con el factor, o bien que por el nivel de desegregación que hemos empleado, el factor no haya podido configurarse con suficiente fuerza. De cualquier manera, el porcentaje de varianza explicado por los tres factores hallados (60\%), deja un amplio margen a otros componentes de la diferenciación residencial del barrio, uno de los cuales puede ser aquel.

\section{DELIMITACIÓN DE ÁREAS SOCIALES}

\subsection{Representación de los tres Factores}

En las figuras 3,4 y 5 se cartografian las puntuaciones factoriales de los tres factores extraídos en el análisis llevado a cabo sobre las 12 variables sociodemográficas seleccionadas.

El primer factor, Urbanización o No Familismo, alcanza los valores positivos más elevados en el Suburbio de Alcalá, gran parte de la Ampliación y algunas manzanas de la promoción de la Concepción. Con valores positivos débiles destacan el Sector Alcalde López Casero, las restantes manzanas de la Ampliación, el Barrio Baterías y gran parte de la Concepción y del Sector José del Hierro. Todas estas áreas se caracterizan por contar con una mayor porporción (a escala del conjunto del barrio) de hogares sin núcleo familiar y mujeres activas, y menor de 

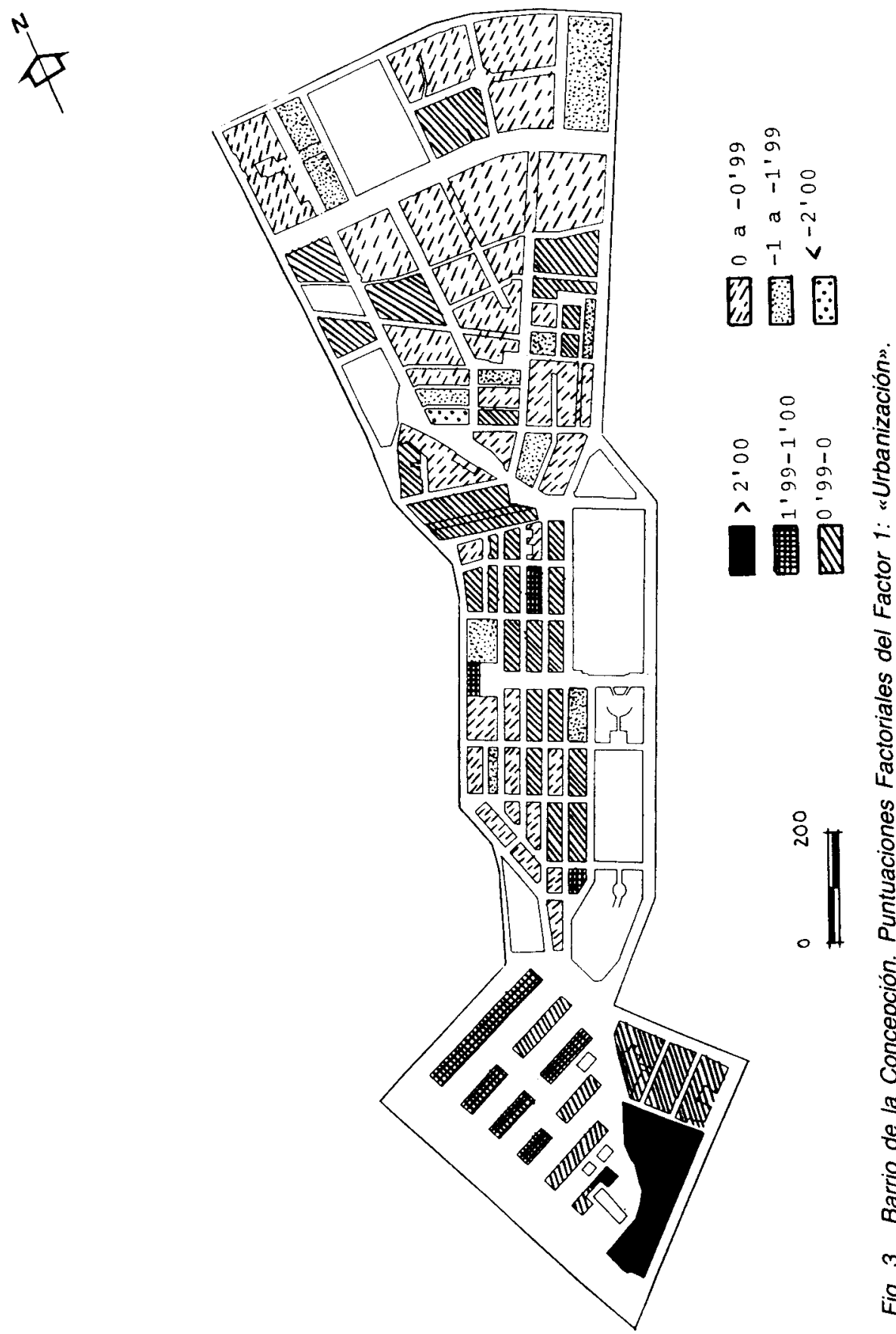

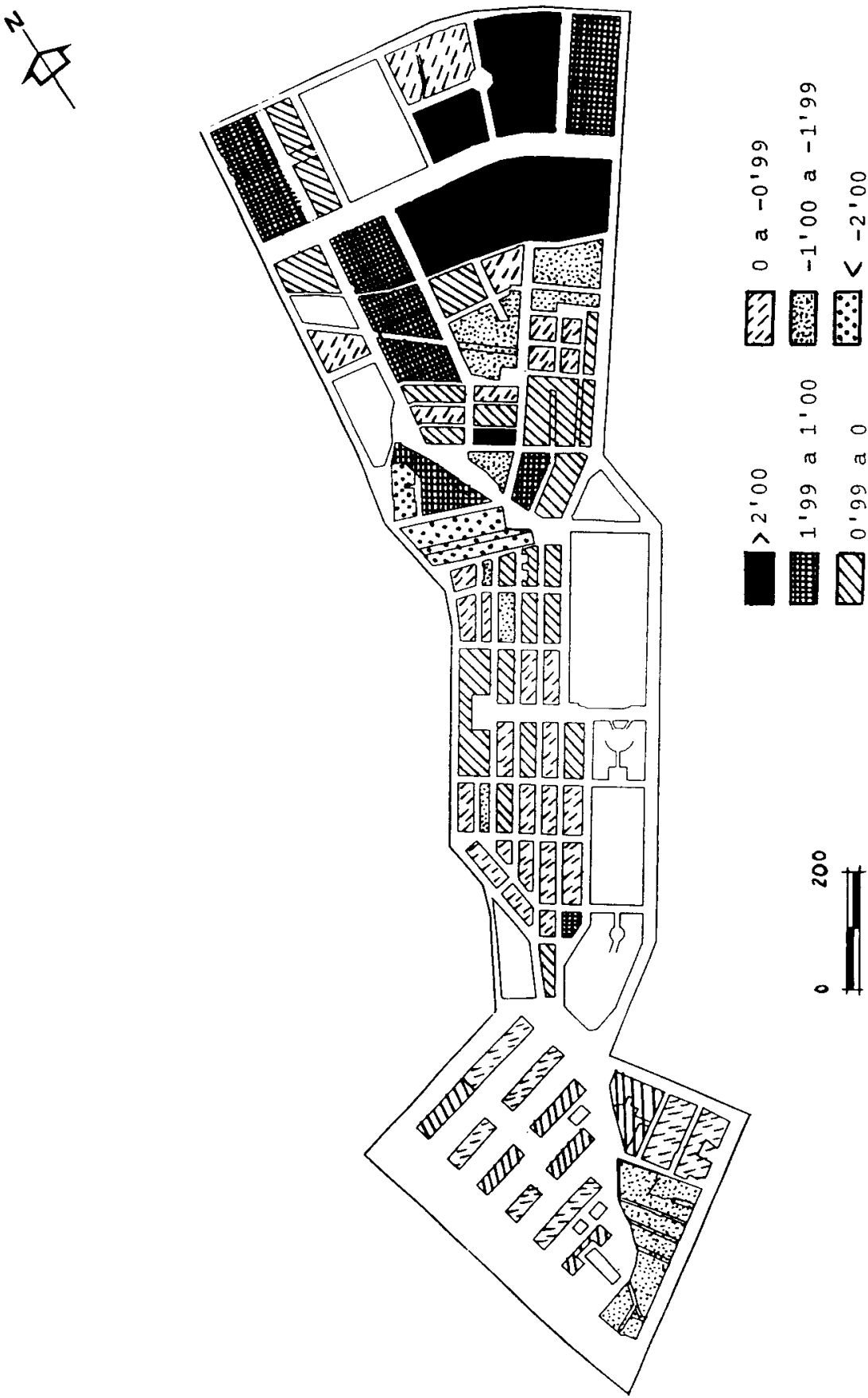

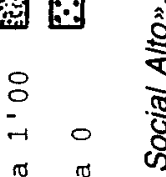

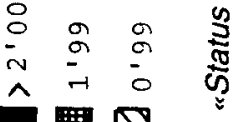

澧

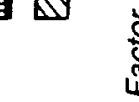

¿ั

ఫ)

$\frac{\mathscr{2}}{\frac{\pi}{2}}$

¿

: 

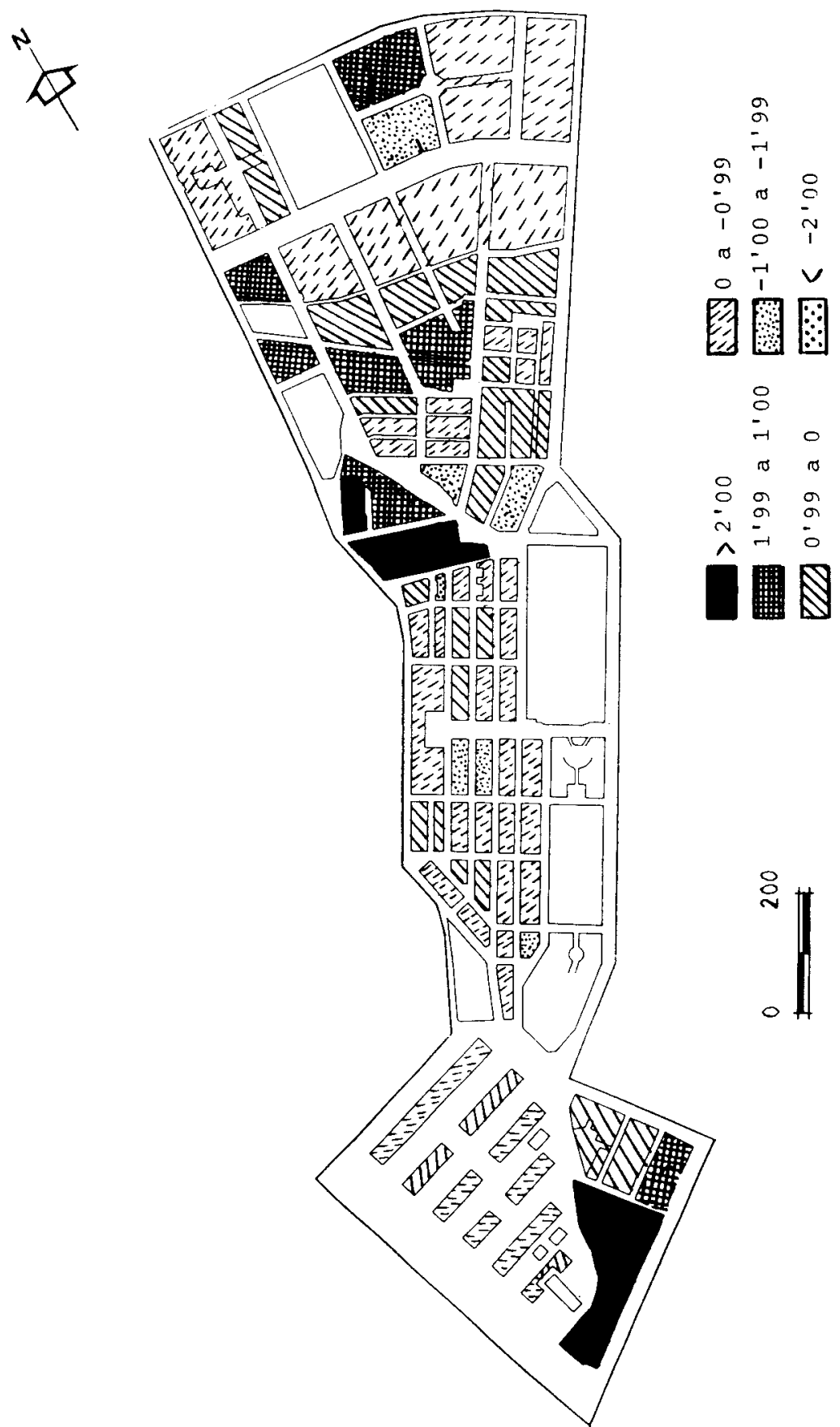

$\stackrel{\circ}{2}$

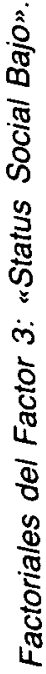

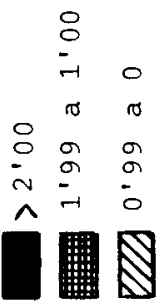

ญ

:

ญ्ष

ঠ

(I)

o

$\frac{2}{\infty}$

is

i̊ 
población infantil y familias amplias. Las familias compuestas por cinco y más miembros, donde abunda la población en edades comprendidas entre 0 y 15 años, son la característica más importante de las áreas cuyas puntuaciones factoriales son negativas, localizadas sobre todo en las zonas de edificación abierta de la mitad oriental del barrio: la Ciudad Lineal, el Sector Intermedio y la colonia San Vicente, en algunas de cuyas manzanas se dan los valores negativos más elevados del factor.

El Factor 2 refleja los grupos de más elevado statuts social del barrio, fundamentalmente profesionales técnicos y patronos con asalariados, titulados medios y superiores o población con un nivel de estudios de bachillerato superior, asi como hogares que cuentan con servicio doméstico. El área de más elevada puntuación en el factor es la Ciudad Lineal, donde destacan, sobre todo, las manzanas dominados por el tipo edificatorio multifamiliar de reciente construcción. Destacan también manzanas de la colonia San Vicente y la Zona Intermedia. En el extremo opuesto, con los valores negativos más altos se sitúan el Barrio Baterías, el Suburbio de Alcalá y algunas manzanas que engloban edificios antiguos del Sector José del Hierro y la Zona Intermedia. El Sector Alcalde López Casero, la Ampliación y la Concepción, tienen valores bajos, tanto positivos, como negativos.

El Factor 3 refleja con valores positivos las manzanas dominadas por población de bajo status social, determinado por la mayor proporción de trabajadores manuales, personas analfabetas o cuyo nivel de instrucción es la primaria incompleta y mínima proporción de personas con un nivel de instrucción que alcance el bachillerato superior. El Barrio Baterías aparece con el valor positivo más destacado (por encima de tres), seguido del suburbio de Alcalá. Ambas «bolsas residuales" suponen, de nuevo, los casos más extremos. En el siguiente umbral figura una manzana del Sector Alcalde López Casero y algunas de la Zona Intermedia y la Ciudad Lineal, cuya trama no ha sido renovada modernamente, además de la manzana del Barrio Baterías que no se ajusta al modelo ni a la edad de esta barriada. En las promociones de la Ampliación y la Concepción, en los sectores Alcalde López Casero y José del Hierro, en la Colonia San Vicente y en la Ciudad Lineal, dominan los valores positivos o negativos débiles.

\subsection{Las Áreas Sociales del Barrio de la Concepción}

En la figura 6 se representan las observaciones sobre un plano tridimensional, donde en cada eje figuran las puntuaciones de cada uno de 
Delimitación de áreas sociales en el Barrio de la Concepción. Un ensayo ...

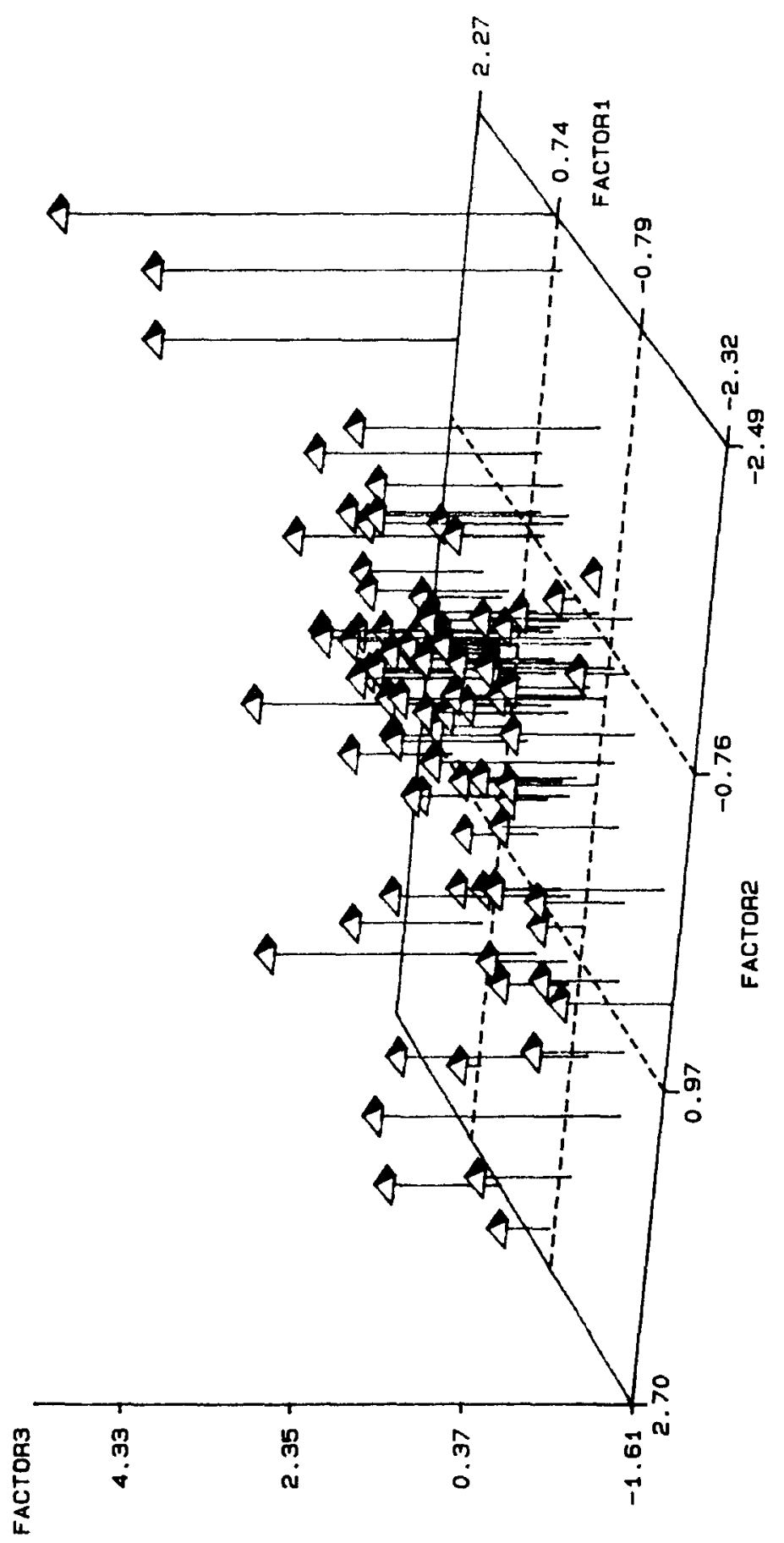

207 
los tres factores. En la parte central del gráfico se agrupan la mayor parte de los casos, que cuentan con valores parecidos en los tres factores. Fuera de este grupo central se observan otros pequeños subgrupos, compuestos por un reducido número de observaciones, que quedan individualizadas por situarse en los extremos de alguno de los ejes. En términos generales, a la derecha del grupo central se sitúan las observaciones caracterizadas por un bajo status social y a la izquierda las de status social más elevado. Las observaciones que destacan en altura, corresponden a los estratos sociales más bajos. Las que se sitúan al fondo del plano se caracterizan por un alto nivel de urbanización y las más próximas, por un elevado grado de familismo.

La representación gráfica de los factores en un plano tridimensional resulta de gran utilidad a la hora de agrupar observaciones y delimitar áreas homogéneas. En este sentido, viene a cumplir la misma función que el análisis de Cluster.

Una vez identificadas cada una de las observaciones según los tres ejes, se clasificaron los casos en seis grupos, que corresponden a otros tantos tipos de Áreas Sociales diferenciadas. En la figura 7 aparecen estas agrupaciones, representadas con distintos símbolos, para facilitar su interpretación. Los tipos delimitados se cartografían en la figura 8. En la cartela, junto a cada trama, se ha representado el símbolo distintivo del grupo que aparece en el gráfico.

Las Áreas Sociales delimitadas son las siguientes:

A) Área de status social inferior y alto grado de urbanización: comprende el Barrio Baterías y el suburbio de Alcalá. Constituyen las áreas de más fuerte especificidad del barrio, que en todos los análisis aparecen como observaciones muy distanciadas de las demás.

B) Manzanas de bajo status social y nivel medio-bajo de urbanización: las observaciones aquí incluidas no llegan a configurar un área, apareciendo de manera aislada, sobre todo en la promoción de la Concepción, la Zona Intermedia y los sectores José del Hierro y Alcalde López Casero. En estas tres últimas zonas se corresponden con manzanas donde predomina la edificación antigua y que, en algunos casos, cuentan con viviendas de tipo marginal.

C) Área de status social contrastado y elevado nivel de urbanización: está configurada únicamente por tres manzanas correspondientes a la Zona Intermedia y a la Colonia San Vicente.

D) Área de alto status social y bajo grado de urbanización: comprende algunas manzanas de la Ciudad Lineal y la Colonia San Vicente. 
Delimitación de áreas sociales en el Barrio de la Concepción. Un ensayo ..
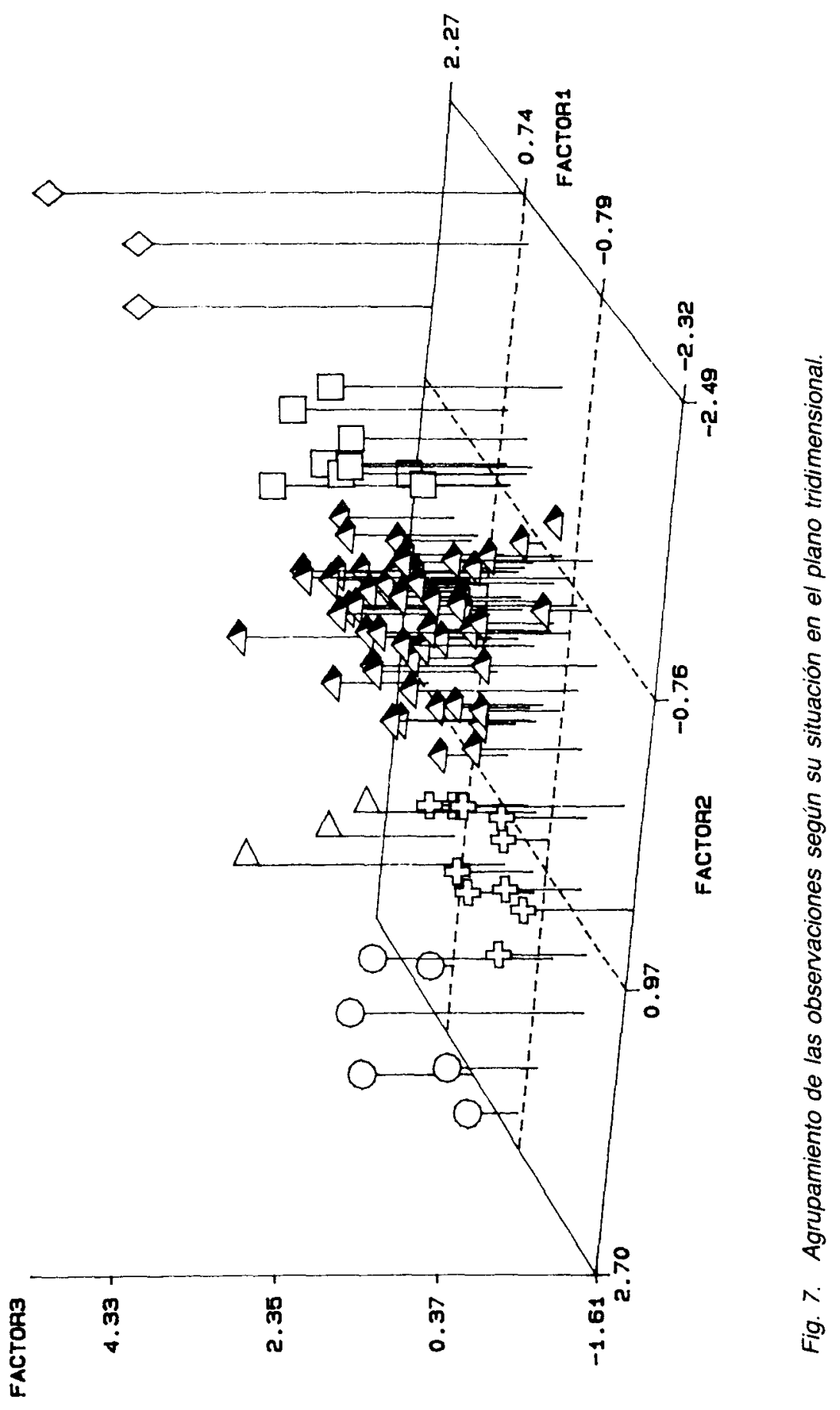

209 
2

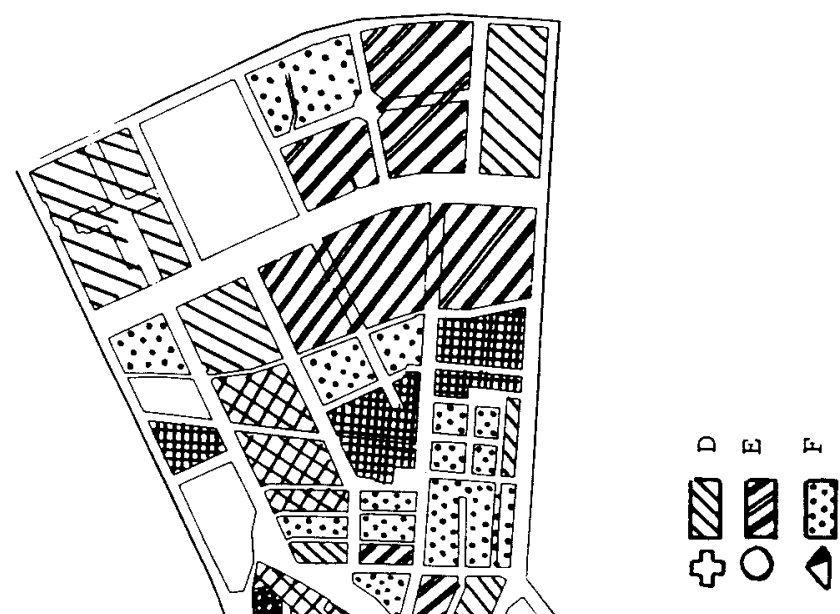

๔ ๓ 0 क

鹿递

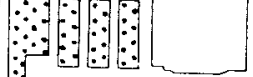

$\diamond \square \triangle$
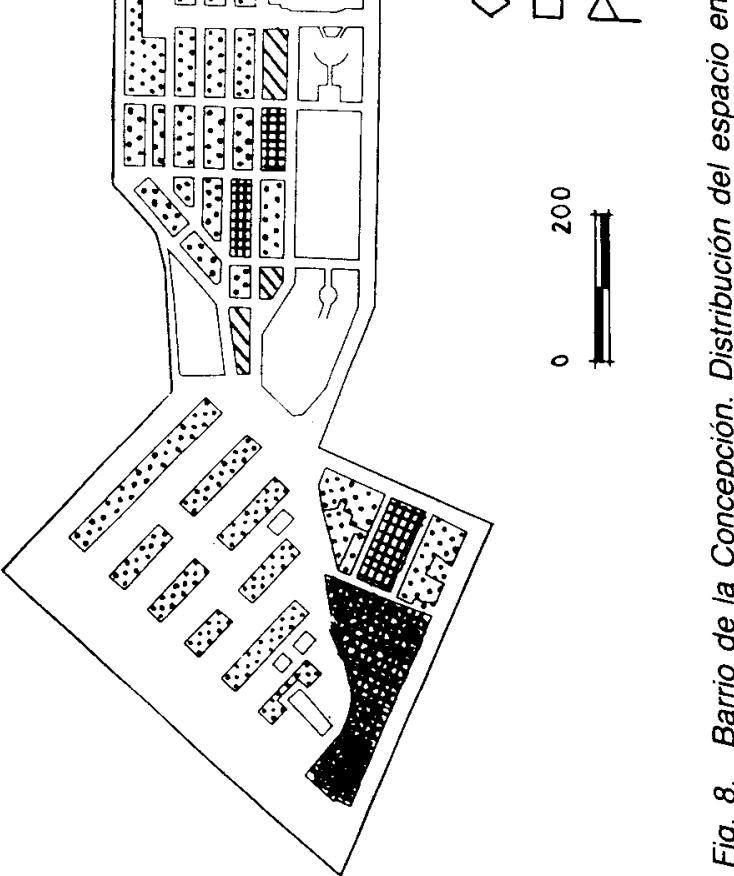
Este tipo está también presente en la promoción de la Concepción y en la manzana de construcción más reciente del Sector José del Hierro.

E) Área de status social superior y nivel medio-bajo de urbanización: queda representada únicamente en la Colonia San Vicente y en algunas de las manzanas de la Ciudad Lineal donde domina el tipo de edificación abierta multifamiliar, de construcción reciente.

F) Área de status social medio y niveles medio-altos de urbanización: comprende 52 unidades espaciales (el $61 \%$ de las observaciones), siendo el tipo dominante en las promociones de la Ampliación (donde quedan incluidas la totalidad de las manzanas) y la Concepción, y estando bastante representado en los sectores Alcalde López Casero y José del Hierro y en la Colonia San Vicente. Conforma el tipo sociodemográfico central que dá el carácter general al barrio y en torno al cual se individualizan las otras áreas.

En resumen, el Barrio de la Concepción presenta una cierta uniformidad en base a un status social de tipo medio y un nivel de urbanización medio-alto. Ello significa que la mayor parte del espacio urbano que estudiamos y del que son su exponente más claro las promociones de la Ampliación y la Concepción, tiene una población en la que dominan los técnicos y el personal administrativo y comercial $^{3}$, donde las familias actuales no están formadas por muchos miembros, existe un número relativamente importante de hogares sin núcleo familiar y una proporción bastante crecida de mujeres en la fuerza laboral.

Dentro de esta relativa homogeneidad, a ambos lados de este tipo sociodemográfico más común, están representados dos polos opuestos de la escala social: un grupo de status social bajo, que se localiza en las "bolsas residuales" de las Baterias y zona expropiada de Alcalá —donde dominan los trabajadores manuales con un bajo o nulo nivel de instrucción-; y un grupo de status social alto, localizado fundamentalmente en las áreas de reciente renovación de la trama urbana, donde domina el tipo de edificación abierta multifamiliar y en áreas aun bien conservadas de viviendas unifamiliares del tipo de ciudad jardín.

Los contrastes más bruscos a nivel espacial, se producen entre los sectores occidental-central del barrio y el sector oriental y dentro de este

${ }^{3}$ En la primera matriz de correlaciones y los dos primeros análisis factoriales, basados en 23 y 17 variables, respectivamente, se pudo comprobar la conexión del personal administrativo y comercial con las variables mujeres activas y hogares sin nucleo y su correlación negativa con las familias amplias y menores de 15 años. 
último sector. En la base de la fuerte heterogeneidad de esta amplia área se encuentra, como factor fundamental -imposible de medir y, por tanto, no susceptible de aislar matemáticamente - el grado de renovación de la trama urbana: el caos urbanístico, tipológico y de edad y calidad de construcción, se traduce en una yuxtaposición de unidades aisladas representativas de tipos sociales de muy alto, alto, medio y bajo status y con unos niveles de urbanización también dispares.

La Ampliación de la Concepción resulta ser el área de mayor cohesión interna ${ }^{4}$, pudiéndose considerar como "vencindario homogéneo", entendido como "grupo territorial distinto, en virtud de sus características físicas específicas y de las características sociales específicas de sus habitantes" (GLASS, R.; en BLOWERS, A. 1975). Su característica más clara es un elevado nivel de urbanización, que destaca dentro del barrio - si descontamos el suburbio de Alcalá-. Es el área donde, por tanto, la proporción de hogares sin núcleo, adultos que viven solos, personal administrativo y comercial y mujeres activas, es más elevada de todo el barrio. En el extremo opuesto en el eje y también en el espacio, la zona oriental se caracteriza por su bajo grado de urbanización, siendo más frecuente las familias amplias y la población infantil. El centro de ambos polos opuestos puede ser, en términos generales, la promoción de la Concepción, que se mantiene en niveles medios en este factor, posiblemente debido a su mayor antigüedad, que hace que las familias se hayan reducido por emancipación de los hijos mayores y donde los hogares sin núcleo familiar no son tan numerosos como en la otra promoción.

En la base de la mayor cohesión interna y homogeneidad de la Ampliación existe, además otro factor: el volumen de población con que cada manzana cuenta. El reducido volumen de la mayor parte del resto de las manzanas del barrio es, por otra parte, un factor fundamental a la hora de comprender algunos de los contrastes que se aprecian dentro de áreas que resultaban morfológicamente homogéneas: los casos particulares tienen tal peso que modifican la caracterización del conjunto de la unidad de análisis.

Con todo, los resultados de los análisis multivariantes llevados a cabo sobre las 85 unidades espaciales, a nivel máximo de desagregación, del Barrio de la Concepción, nos parecen totalmente válidos y significativos de la realidad sociodemográfica de la zona de estudio.

${ }^{4}$ En un análisis Cluster realizado en base a 23 variables sociodemográficas, todas las manzanas de la Ampliación se unian en el mismo cluster, formado a 0,30 del centro de gravedad, mostrando ser el área más homogénea. 


\section{CONCLUSIONES}

Del trabajo llevado a cabo, podemos deducir las siguientes conclusiones:

1. Desde el punto de vista metodológico, el análisis a una escala de desagregación espacial máxima, hace que los índices de correlación y las cargas de los factores tengan valores más bajos que a otras escalas mayores. Ello confirma la tesis de JIMÉNEZZ BLASCO, B. C., (1986). Asimismo, la estructura factorial resulta más compleja a este nivel, si bien la solución factorial se simplifica al ir eliminando las variables menos significativas.

2. La estructura factorial final resulta coherente.

3. Las áreas sociales delimitadas por los factores obtenidos, muestran una estrecha relación con las áreas morfológicas. Los sectores urbanos modernos, de buena calidad arquitectónica, son lugar de residencia de grupos sociales de alto status. Por su parte, los grupos de bajo status social se relacionan con el paisaje urbano degradado de casas antiguas y de mala calidad. Asimismo, existe un contraste entre las áreas edificadas más densamente pobladas y las de edificación abierta y unifamiliar, en relación con factores como el urbanismo y el familismo. La elevada densidad de viviendas de la Ampliación, propicia la proliferación de hogares sin núcleo familiar, el anonimato de la gran ciudad y el aislamiento social, propio de un estilo de vida "consumista" o "carrerista", frente a la colonia San Vicente o la Ciudad Lineal, donde la familia es protagonista en el estilo de vida de las personas.

\section{RESUMEN}

En el presente artículo se realiza un análisis de Ecología Factorial de un barrio de Madrid, a nivel máximo de desagregación espacial. Se comparan los resultados con los obtenidos en otros estudios sobre Madrid y se comprueba la relación entre las áreas morfológicas del barrio y las áreas sociales deducidas del análisis. 


\section{ABSTRACT}

In this paper a Factorial Ecology analysis of a neighbourhood in Madrid, in a highest level of spacial segregation, is performed. A comparison between our results and those from other studies on Madrid, is made. The relationship between morphological and social areas in our neighbourhood, is also stablished. 


\section{BIBLIOGRAFIA}

Aguilera ARILla, M. J. 1988: «Diferenciación del espacio social de Alcorcón", Estudios Geográficos t. XLIX, núm. 190, enero-marzo, Madrid, págs. 5-28.

Blowers, A. 1977: «The Neighbourhood. Exploration of a concept», en The City as a Social Problem. Unit 7. The Open University.

JIMÉNEZ BLASCO, B. C. 1984: “Aproximación metodológica al estudio de la diferenciación residencial urbana en Madrid", Anales de Geografía de la Universidad Complutense núm. 4. Madrid, págs. 167-184.

JiménEZ BLASCO, B. C. 1988: Estudio de la diferenciación residencial en la ciudad de Madrid. Ed. de la Universidad Complutense de Madrid. Colección Tesis Doctorales núm. 315/88. 691 págs.

LORA-TAMAYO D'OCÓN, G. 1990: “Una revisión de los estudios sobre diferenciación sociodemográfica referidos a la ciudad de Madrid", Espacio, Tiempo y Forma, Serie IV Geografia, t. 3. Madrid, págs. 5592.

SWEESTER, F. L. 1965: "Factorial Ecology. Helsinki, 1960", Demography, 2. Págs. 372-386.

TIMMS, D. 1976: «El Mosaico Urbano. Hacia una teoría de la diferenciación residencial', IEAL Col. Nuevo Urbanismo, núm. 21. Madrid, 458 páginas. 